\title{
BASIC TOPOLOGICAL STRUCTURES OF THE THEORY OF ORDINARY DIFFERENTIAL EQUATIONS
}

\author{
V. V. FILIPPOV \\ Chair of General Topology and Geometry, Department of Mathematics \\ Moscow State University, 119899, Moscow V-234, Russia \\ E-mail: fil@sci.math.msu.su
}

If you open any book on the theory of ordinary differential equations (TODE) you can observe that almost every proof in the TODE uses the continuity of the dependence of a solution of the Cauchy problem on initial data and parameters of the right-hand side.

But the classical TODE has few theorems assuring this continuity. In general they suppose the continuity of the right-hand side.

We can note a trivial

EXAmPLE 0.1. Let $g(t, y)$ be a continuous function. The dependence of solutions of the equations $y^{\prime}=g(t, y)+\alpha\left(t^{2}+y^{2}+\alpha^{2}\right)^{-1}$ on the parameter $\alpha$ is continuous though the right-hand side is not continuous at the point $t=y=\alpha=0$.

So we meet the problem to describe a topological structure adequate to the idea of the continuity of the dependence of solutions on parameters of the right-hand side of the equation.

Solving this problem we develop a new large working theory which gives a natural framework of topological contents of the TODE. The theory deals with equations of classical types, with equations having various singularities in the right-hand sides, with differential inclusions etc.

Some account of the theory may be found in [7] (see also the Chapter IX in the book [4] and the book [8]). You can find there proofs of many results of this survey (see also [9]-[32], [35] for other details).

The theory of Sections 1-6 is rather old. The author is very grateful to Professors J. Mawhin and L. Górniewicz for valuable contacts which have greatly stimulated results of Section 7. He is also thankful to B. S. Klebanov for discussing this paper.

The paper is in final form and no version of it will be published elsewhere. 
1. The space of partial mappings. Given an ordinary differential equation, we call its solutions functions defined on different domains. On the other hand, traditional methods of investigation of equations are based on topological properties of functions which are defined on a fixed domain. For instance, constantly we use the uniform convergence of sequences of solutions but this convergence is defined only if the solutions in question have common domain of definition.

The noted situation causes an impossibility to use functional methods everywhere. If we come across an equation where functional methods are not applicable, we begin to look for what is the obstacle. In other words, we begin to study singularities.

Here we follow just this way. First we define a topological structure which can help us. Up to my knowledge the first person who considered a structure of this type was a Polish mathematician S. K. Zaremba [43] (see also [36]). K. Kuratowski in [38] and [39] approached analogous notions in another respect.

Let $X$ and $Y$ be Hausdorff topological spaces, and $A$ a closed subset of the space $X$. Any continuous mapping $f: A \rightarrow Y$ will be called a partial mapping from $X$ to $Y$. Let us denote by $C_{v}(X, Y)$ the set of all partial mappings from $X$ to $Y$, and by $C_{c v}(X, Y)$ the set of all partial mappings from $X$ to $Y$ having compact domains. The topology of this spaces is uniquely determined by the condition that the injective map $\operatorname{Gr}: C_{v}(X, Y) \rightarrow$ $\exp (X \times Y)$, which associates with a function $\varphi \in C_{v}(X, Y)$ its $\operatorname{graph} \operatorname{Gr}(\varphi) \in \exp (X \times Y)$, is an embedding [40]. (Here $\exp X$ denotes the space of all nonempty closed subsets of $X$ with the Vietoris topology and $\exp _{c} X$ denotes the space of all nonempty compact subsets of $X$.)

Let us start with listing some technical properties of these notions which are important for their applications in the TODE.

Let $H$ be a closed subset of the space $X$. We can consider two topologies on the set $C(H, Y)$ of all continuous mappings from $H$ to $X$. The first one is the compact-open topology. We shall denote the corresponding topological space by $C_{b}(H, Y)$. We obtain the second one by considering the set as a subspace of the space $C_{v}(X, Y)$. In this case we shall keep the notation $C(H, Y)$.

Theorem 1.1. Let $H \in \exp X$. Then the identity mapping $C(H, Y) \rightarrow C_{b}(H, Y)$ is continuous.

TheOrem 1.2. Let $H \in \exp _{c} X$. Then the identity mapping $C(H, Y) \rightarrow C_{b}(H, Y)$ is a homeomorphism.

Theorem 1.2 shows that the topological structure of the space $C_{c v}(X, Y)$ generalizes the compact-open topology on the set of functions having a fixed domain.

If the spaces $X$ and $Y$ are metric, then the topology of the space $\exp (X \times Y)$ is metrizable by the corresponding Hausdorff metric. So are the topologies of the spaces $\operatorname{Gr}\left(C_{c v}(X, Y)\right)$ and $C_{c v}(X, Y)$.

If the spaces $X$ and $Y$ are complete metric spaces, then the topology of the space $\exp (X \times Y)$ is metrizable by the corresponding complete Hausdorff metric. But in general case the set $\operatorname{Gr}\left(C_{c v}(X, Y)\right)$ is not closed in the space $\exp (X \times Y)$ and hence this way does not give us a complete metric on the space $C_{c v}(X, Y)$. However we have 
Theorem 1.3. Let the space $Y$ be metrizable. Then the set $\operatorname{Gr}\left(C_{c v}(X, Y)\right)$ is a $G_{\delta}$ subset of the space $\exp (X \times Y)$.

It follows from Theorem 1.3 that if the spaces $X$ and $Y$ are complete metric spaces, then the space $C_{c v}(X, Y)$ is metrizable by a complete metric.

Let us note one more relation of the space $C_{c v}(X, Y)$.

TheOrem 1.4. The mapping $\pi: C_{v}(X, Y) \rightarrow \exp X$ which associates with a function $\varphi \in C_{v}(X, Y)$ its domain is continuous. If the space $Y$ is a singleton, then the mapping $\pi$ is a homeomorphism.

The importance of the uniform convergence for the Analysis is ensured by the fact that the mapping which associates with any element $(z, t)$ of the product $C(T, Y) \times T$ the value $z(t) \in Y$ is continuous for the corresponding topology on the set $C(T, Y)$. The space $C_{v}(X, Y)$ has a similar property.

Let $\varphi$ be a filter on a set $A$. A family $\left\{M_{\alpha} \mid \alpha \in A\right\}$ (with a fixed filter $\varphi$ on $A$ ) is said to be a generalized sequence. Various versions of the notion of its limit are defined in a natural way. We shall keep the usual notation.

THeOREM 1.5. Let $X$ and $Y$ be Hausdorff topological spaces, let the space $X$ be normal, and let generalized sequences $\left\{z_{\alpha} \mid \alpha \in A\right\} \subseteq C_{v}(X, Y)$ and $\left\{D_{\alpha} \mid \alpha \in A\right\} \subseteq \exp X$ converge by filter $\varphi$ to $z \in C_{v}(X, Y)$ and $D \in \exp X$ respectively. Suppose that $D_{\alpha} \subseteq \pi\left(z_{\alpha}\right)$ for each $\alpha \in A$. Then $D \subseteq \pi(z)$ and the generalized sequence $\left\{\left.z_{\alpha}\right|_{D_{\alpha}} \mid \alpha \in A\right\}$ converges by filter $\varphi$ to $\left.z\right|_{D}$.

On the other hand we have

Theorem 1.6. Let $X$ and $Y$ be Hausdorff topological spaces, $\left\{z_{\alpha} \mid \alpha \in A\right\} \subseteq C_{v}(X, Y)$, $z \in C_{v}(X, Y),\left\{D_{\alpha} \mid \alpha \in A\right\},\left\{E_{\alpha} \mid \alpha \in A\right\} \subseteq \exp X, D_{\alpha} \cup E_{\alpha} \subseteq \pi\left(z_{\alpha}\right)$ for each $\alpha \in A, D, E \in \exp X$ and generalized sequences $\left\{\left.z_{\alpha}\right|_{D_{\alpha}} \mid \alpha \in A\right\}$ and $\left\{\left.z_{\alpha}\right|_{E_{\alpha}} \mid \alpha \in\right.$ $A\}$ converge by filter $\varphi$ in the space $C_{v}(X, Y)$ to $\left.z\right|_{D}$ and $\left.z\right|_{E}$ respectively. Then the generalized sequence $\left\{\left.z_{\alpha}\right|_{D_{\alpha} \cup E_{\alpha}} \mid \alpha \in A\right\}$ converges by filter $\varphi$ in the space $C_{v}(X, Y)$ to $\left.z\right|_{D \cup E}$.

Both theorems have many applications in proofs of results of our theory.

One of the main properties of the space $C_{v}(X, Y)$, where the spaces $X$ and $Y$ are metric, is the possibility to obtain a suitable version of Arzela theorem. The notion of the equicontinuity keeps its meaning in this case. For $M \subseteq X \times Y$ and $Z \subseteq C_{v}(X, Y)$ let us denote by $Z_{M}$ the set $\{z \mid z \in Z, \operatorname{Gr}(z) \subseteq M\}$.

TheOREM 1.7. Let $X$ and $Y$ be metric spaces. Then every compact subset of the space $C_{v}(X, Y)$ is equicontinuous.

TheOREM 1.8. Let $K$ be a compact subset of the product of metric spaces $X$ and $Y$, and let a sequence $\alpha=\left\{z_{k} \mid k=1,2, \ldots\right\} \subseteq\left(C_{v}(X, Y)\right)_{K}$ be equicontinuous. Then there exists a convergent (in $\left.C_{v}(X, Y)\right)$ subsequence of the sequence $\alpha$.

Below the case of $X=\mathbb{R}$ and $Y=\mathbb{R}^{n}$ will be considered only. Let us denote by $C_{s}\left(\mathbb{R} \times \mathbb{R}^{n}\right)$ the set of all mappings from $C_{v}\left(\mathbb{R} \times \mathbb{R}^{n}\right)$ having compact connected domains (we shall consider singletons as degenerate segments; so the elements of $C_{s}\left(\mathbb{R} \times \mathbb{R}^{n}\right.$ ) have 
segments as domains $)$. Let $C_{s}(M)=\left(C_{s}\left(\mathbb{R} \times \mathbb{R}^{n}\right)\right)_{M}$ for $M \subseteq \mathbb{R} \times \mathbb{R}^{n}$. The set $C_{s}(M)$ is closed in the space $\left(C_{c v}\left(\mathbb{R}, \mathbb{R}^{n}\right)\right)_{M}$.

2. Basic properties of solution spaces and the Cauchy problem. Aiming to define an adequate topological structure, let us start with a general definition of solution which may be suitable in all particular cases below.

Let $U$ be a subset of the product $\mathbb{R} \times \mathbb{R}^{n}$. We shall consider differential equations $y^{\prime}=f(t, y)$ or their generalized versions (inclusion) $y^{\prime} \in f(t, y)$ having right-hand sides defined on the set $U$. A function $z \in C_{s}(U)$ will be called a solution of the equation if

a) the segment $\pi(z)$ is not degenerate, the function $z$ is generalized absolutely continuous and $z^{\prime}(t)=f(t, z(t))\left(\right.$ resp. $\left.z^{\prime}(t) \in f(t, z(t))\right)$ for almost all $t \in \pi(z)$,

or

b) $|\pi(z)|=1 \quad$ (in other words $\pi(z)$ is a singleton).

The set of all solutions to the equation $y^{\prime}=f(t, y)$ (resp., inclusion $y^{\prime} \in f(t, y)$ ) will be denoted by $D(f)$. For $M \subseteq U$ let $D(f, M)=\left.(D(f))\right|_{M}$.

In these definitions we do not fix domains of functions. We consider them all together. So the solution set $D(F)$ lies in the space $C_{s}(U)$.

In what follows $U$ will be open in $\mathbb{R} \times \mathbb{R}^{n}$.

For equations with continuous right-hand sides our definition gives only continuously differentiable solutions. For equations with right-hand sides satisfying the Carathéodory conditions this definition gives Carathéodory solutions etc.

Our generalization of the notion of solution can be applied in situations which are very close to the classical theory.

EXAMPLE 2.1. Let $g(t, y)$ be a function which is continuous everywhere but one point. The properties of so defined solutions of the equation $y^{\prime}=g(t, y)$ are the same as those of solutions of the equations studied in the classical theory. This case is not covered by the Carathéodory theorem because we need not have here an integrable majorant.

Note that our approach is antipodal to the approach suggested by A. F. Filippov in [33] (see also [34]). He changes the equation $y^{\prime}=f(t, y)$ under consideration with discontinuities in the space variables by an inclusion $y^{\prime} \in F(t, y)$ related in a natural way to the equation $y^{\prime}=f(t, y)$, and he calls a solution of the equation every solution of the inclusion without any supplementary consideration. We deal with the most natural possible notion of solution. This two ways can give different set of solutions.

The levels of the TODE correspond to the levels of the theory of integration. The TODE with continuous right-hand side corresponds to the integral of Riemann. The TODE under the conditions of Carathéodory corresponds to the integral of Lebesgue. The application of our theory gives us a level corresponding to the integral of Denjoy. This level has been already reached in Example 2.1.

The idea that various properties of solutions depend one on another is not too original. There exist some results about the properties without direct reference to the equation. But we shall go further. One of our tools will consist of precise analysis of such relations. 
Let us highlight some basic properties of solution set $Z$ :

$$
\text { if } z \in Z \text { and a segment } I \text { lies in } \pi(z) \text {, then }\left.z\right|_{I} \in Z \text {, }
$$

$$
\begin{gathered}
\text { if } z_{1}, z_{2} \in Z, I=\pi\left(z_{1}\right) \cap \pi\left(z_{2}\right) \neq \emptyset \text { and }\left.z_{1}\right|_{I}=\left.z_{2}\right|_{I} \text { then the function } \\
z(t)= \begin{cases}z_{1}(t) & \text { if } t \in \pi\left(z_{1}\right), \\
z_{2}(t) & \text { if } t \in \pi\left(z_{2}\right)\end{cases} \\
\text { (defined on the segment } \left.\pi\left(z_{1}\right) \cup \pi\left(z_{2}\right)\right) \text { belongs to the set } Z,
\end{gathered}
$$

(p) the set $Z$ contains all functions from $C_{s}(U)$ defined on singletons and if a function $z \in C_{s}(U)$ is defined on a segment $[a, b]$ where $a<b$ and $\left.z\right|_{I} \in Z$ for each segment I lying in $] a, b[$ then $z \in Z$,

(c) the set $Z_{K}$ is compact for every $K \in \exp _{c} U$,

(e) for each point $(t, y)$ of the set $U$ there exists a function $z \in Z$ such that $t \in\langle\pi(z)\rangle$ and $z(t)=y(\langle M\rangle$ denotes the interior of $M)$,

(u) if $z_{1}, z_{2} \in Z, \pi\left(z_{1}\right)=\pi\left(z_{2}\right)$ and $z_{1}(t)=z_{2}(t)$ for some $t \in \pi\left(z_{1}\right)$, then $z_{1}=z_{2}$.

It is easy to see that properties (1), (2) and (p) follow directly from our definitions. Conditions (e) and ( $\mathrm{u}$ ) correspond to the existence theorem and the uniqueness theorem. Condition (c) corresponds to a weak version of the condition of the continuity of the dependence of solutions of the Cauchy problem on initial values.

Next natural step should be to develop a theory having noted properties as axioms. Some such investigations have old history, see [43], [36]. New theorems following this approach may be found below. But if we have no possibilities to prove this properties out the classical TODE, the importance of such results stays uncertain. So having the purpose to create a working valuable axiomatic TODE we must think about developing of new non-classical methods of proving the basic properties. We shall do it.

One of important aspects of the properties is their local character. This implies new strong possibilities of studying singularities of equations. In such studies we now do not need to refer constantly to properties of equations. We obtain an opportunity to prove the inessentiality of singularities by steps. Suppose that for each element $V$ of a family $\gamma$ of open subsets of the set $U$ we have proved that the space $Z_{V}$ is "good" (has no essential singularities). In many cases we can estimate the remainder $U \backslash \cup \gamma$ so that it becomes obvious that the whole space $Z$ is "good" too. Classical results or considerations like just described may be used for proving "goodness" of the spaces $Z_{V}$, where $V \in \gamma$. Examples of such propositions will be given below.

We shall denote by $R^{i}(U)$ the set of all $Z \subseteq C_{s}(U)$ satisfying condition (1) and by $R(U)$ the set of all $Z \subseteq C_{s}(U)$ satisfying conditions (1) and (2). Let $*$ denote an arbitrary set of conditions on subspaces of the space $C_{s}(U)$. The symbol $R_{*}^{i}(U)$ (respectively $R_{*}(U)$ ) will denote the set of all elements of $R^{i}(U)$ (respectively of $R(U)$ ) satisfying all conditions of $*$. The sets $R_{*}^{(i)}(U)$ will be called "classes of solution spaces".

Our language is not traditional for the TODE and first we shall indicate the following relation between the classical situation and ours. 
THEOREM 2.1. Let $Z \in R_{p}(U)$ and for each $K \in \exp _{c}(U)$ and for each segment $I$ of $\mathbb{R}$ the set $Z_{K} \cap \pi^{-1}(I)$ be closed in the space $C\left(I, \mathbb{R}^{n}\right)$ and the set $Z_{K}$ be equicontinuous. Then $Z \in R_{c}(U)$.

Theorem 2.1 gives the possibility to deduce the fulfillment of condition (c) from traditional descriptions of properties of solution sets. So under hypotheses of the theorems of Peano [33], Carathéodory [2] and Davy [3] the solution sets belong to $R_{c e}(U)$. The class $R_{c e}(U)$ is one of the most important classes of solution spaces. Many theorems of the classical theory find here their generalization.

For the class $R_{c e}(U)$ theorems on the continuity of the dependence of a solution of the Cauchy problem on initial data and some other important propositions are true (under the assumption of condition $(\mathrm{u})$ in corresponding cases). So we obtain the possibility of its application out the classical TODE.

The properties of belonging of a solution space to the class $R_{c e}(U)$ is an example of a "good" property.

Proposition 2.1. Let $\gamma$ be a family of open subsets of the set $U,\left.Z\right|_{V} \in R_{c e}(V)$ for each $V \in \gamma$ and $|U \backslash \cup \gamma|=1$. Then $Z \in R_{c e}(U)$.

See Example 2.1. The proof of the statement is easy. We get as its trivial corollary.

Proposition 2.2. Let $\gamma$ be a family of open subsets of the set $U,\left.Z\right|_{V} \in R_{c e}(V)$ for each $V \in \gamma$. Let the set $U \backslash \cup \gamma$ be at most countable. Then $Z \in R_{c e}(U)$.

(Proof is based on the following classical proposition of general topology: every countable complete metric space has an isolated point. Then we apply Proposition 2.1. I do not think that the proof of the corresponding statement for the classical TODE without suggestions of our theory is so easy.)

Above we have dealt only with solutions defined on closed segments. Our real purpose there was just to define what must mean words "solution spaces".

For $Z \in R(U)$ we shall denote by $Z^{+}$(respectively by $Z^{-}$) the set of all functions $z:\left[a, b\left[\rightarrow \mathbb{R}^{n}\right.\right.$ (respectively the set of all functions $\left.\left.z:\right] a, b\right] \rightarrow \mathbb{R}^{n}$ ) with arbitrary $a<b$ $( \pm \infty$ may be taken as open ends) satisfying the conditions:

(2.1) $\left.z\right|_{I} \in Z$ for every segment I lying in $\pi(z)$,

(2.2) there is no element in $Z$ extending $z$.

For $Z \in R(U)$ we shall denote by $Z^{-+}$the set of all functions $\left.z:\right] a, b\left[\rightarrow \mathbb{R}^{n}\right.$ with arbitrary $a<b( \pm \infty$ may be taken as open ends) satisfying the condition:

(2.3) $\left.z\right|_{] a, t]} \in Z^{-}$and $\left.z\right|_{[t, b[} \in Z^{+}$for some (and then for each) $\left.t \in\right] a, b[$.

TheOREM 2.3. Let $z \in Z \in R_{c e}(U)$. Then there exists a function from $Z^{-+}$extending the function $z$.

Let $\Omega^{+}(z)=\cap\left\{\left[z(\pi(z) \cap[t, \infty[)] \mid t \in \pi(z)\}\right.\right.$ for $z \in Z^{+} \cup Z^{-+}$.

3. Converging sequences of solution spaces and the space $R^{i}(U)$. Now we shall consider a notion of convergence adequate to the question about the continuity of 
the dependence of a solution of the Cauchy problem on parameters of the right-hand side. Let $U$ be an open subset of the product $\mathbb{R} \times \mathbb{R}^{n}$.

We say that a sequence $\left\{Z_{i} \mid i=1,2, \ldots\right\} \subseteq R^{i}(U)$ converges (in $U$ ) to a space $Z \in R^{i}(U)$ if

(3.1) for any $K \in \exp _{c} U$ and for any sequence $z_{i} \in\left(Z_{i}\right)_{K}\left(i_{1}<i_{2}<\ldots\right)$ there is a subsequence $\left\{z_{i_{m}} \mid m=1,2, \ldots\right\}$ converging to a function $z \in Z$.

Rem ark 3.1. Let $Z \in R^{i}(U)$. The convergence of the sequence $Z_{i} \equiv Z$ to the space $Z$ is equivalent to the condition $Z \in R_{c}^{i}(U)$. Thus results of the study of condition (3.1) can be applied to prove the fulfillment of condition (c).

Properties of this type are known in the TODE. See for instance Theorem I.2.4 in [33]. But they are not so general and complete as our condition is, and I do not know papers where such properties are topics of investigation. In [33] and in other texts where we come across such properties, the authors note them and pass quickly to notions which are charged less by technical details (compare our condition (3.1) with cumbersome and incomplete description in Theorem I.2.4 in [33]).

Our next step will be to define a topology which is adequate to the convergence in (3.1). But we have noted in the remark that the convergence of the stationary sequence is a valuable property of the corresponding solution space. Not every solution space satisfies this condition. On the other hand, in every topological space every stationary sequence converges. Thus we are able to define the topology in question on the set $R_{c}^{i}(U)$ only. In fact here such a topology exists.

In the set $R_{c}^{i}(U)$ the convergence (3.1) corresponds strictly to the following topology. For $M \subseteq U$ and $V \subseteq C_{s}(U)$ let $O\{M, V\}=\left\{Z \mid Z \in R^{i}(U), Z_{M} \subseteq V\right\}$. We shall consider the family $\left\{O\{K, V\} \mid K \in \exp _{c} U, V\right.$ is an open subset of $\left.C_{s}(U)\right\}$ as a subbase of the space $R^{i}(U)$. This condition defines the unique topology on $R^{i}(U)$. We consider the sets $R_{c}(U)$ and $R_{c}^{i}(U)$ as subspaces of so defined space $R^{i}(U)$.

To display the position of this notion, let us consider the equation

$$
y^{\prime}=f(t, y, \alpha),
$$

where the right-hand side depends on a parameter $\alpha \in A$. Then the continuity of the mapping $A \rightarrow R_{c}(U)$ which associates with a parameter $\alpha$ the solution space of the equation $y^{\prime}=f(t, y, \alpha)$, is equivalent to the continuity of the dependence of solutions of equation (3.2) on the parameter $\alpha$ (in a form which does not involve the uniqueness of solutions in general case and in a strict form under the supposition of the uniqueness).

Proposition 3.1. A sequence $\left\{Z_{i} \mid i=1,2, \ldots\right\} \subseteq R^{i}(U)$ converges to $Z \in R_{c}^{i}(U)$ in the space $R^{i}(U)$ if and only if condition (3.1) is fulfilled.

Proposition 3.2. A point $Z \in R_{c}^{i}(U)$ belongs to the closure of a subset $M$ of the space $R^{i}(U)$ if and only if there exists a sequence $\left\{Z_{i} \mid i=1,2, \ldots\right\} \subseteq M$ converging to $Z$.

The spaces $R(U)$ and $R_{c}(U)$ are not $T_{1}$, but our purpose was not to construct any space having nice properties but to find a topology strongly adequate to questions under consideration, what is done. 
The closeness of relations between convergence (3.1) and the topology of the space $R_{c}(U)$ can lead to the idea that we can avoid introducing the topology and consider only the convergence. In fact it is not so. It is very difficult to describe on the level of convergence all needed properties and to prove some relations. In particular the first countability of the space $R_{c}(U)$ is one of its properties enabling applications in the TODE. On the other hand, the existence of topological space $R_{c}(U)$ allows to emphasize easily fundamental properties and relations of solution spaces. For instance, we have

TheOREM 3.1. The subset $R_{c e}(U)$ of the space $R_{c}(U)$ is closed.

The calmness of the last statement can hide its valuable contents. Really this fact is applied in standard proofs of theorems of Peano, Carathéodory and Davy. It now can be used for investigation of equations outside the classical TODE. Let $y^{\prime}=g(t, y)$ be such equation. In various cases the right-hand side $g$ of the equation can be "approximated" in some generalized sense by a sequence $g_{n}$. In our theory there are more possibilities to prove the convergence $D\left(g_{n}\right) \rightarrow D(g)$ than in the classical one. So more equations can be easily investigated. We are able to find new existence theorems or to apply the Theorem 3.1 directly to particular equations.

B. S. Klebanov showed that the set of elements of $R_{c e}(U)$ having the property of Wazewski is closed in $R_{c e}(U)$, see [34].

The fulfillment of the following property for the equation with continuous right-hand side was proved by H. Kneser in [37] (see also [33]):

$(\mathrm{k})$ for each point $(t, y)$ of the set $U$ there exists a number $\varepsilon>0$ such that if $s \in \mathbb{R}$ and $|s-t|<\varepsilon$ then the set $\{z(s) \mid z \in Z, s, t \in \pi(z), z(t)=y\}$ is connected.

TheOREM 3.2. The subset $R_{\text {cek }}(U)$ of the space $R_{c}(U)$ is closed.

In some questions the following homological triviality of solution sets is important:

(h) for each point $(t, y)$ of the set $U$ there exists a number $\varepsilon>0$ such that if $s \in \mathbb{R}$ and $t<s<t+\varepsilon$ then the set $\{z \mid z \in Z, s, t \in \pi(z), z(t)=y\}$ is acyclic.

THEOREM 3.3. The subset $R_{\text {ceh }}(U)$ of the space $R_{c}(U)$ is closed.

Theorem 3.3 gives us a possibility to prove easily the fulfillment of condition $(\mathrm{h})$ in cases which are quite non-trivial for other ways of investigation (see remarks related to condition (3.3) below).

The same technical tools allow to prove that a solution space $Z$ under consideration satisfies the following condition

$$
Z \in\left[R_{\text {ceu }}(U)\right]
$$

which has many important consequences. The real situation is such that the construction of a solution space $Z$ which does not satisfy condition (3.3) requires noticeable additional efforts. Condition (3.3) is satisfied in every natural situation related to equations and inclusions (for equations with complicated right-hand side too).

One of the most important statements about converging sequences of spaces is the following theorem. It looks too technical but has many applications in various situations. The theorem is closely related to J. Yorke's topology [42]. 
TheOrem 3.4. Let a sequence of spaces $\left\{Z_{i} \mid i=1,2, \ldots\right\} \subseteq R_{c e}(U)$ converge to a space $Z \in R_{c e}(U), z_{i} \in Z_{i}^{-+}, t_{i} \in \pi\left(z_{i}\right)$ and the sequence of points $\left\{\left(t_{i}, z_{i}\left(t_{i}\right)\right) \mid i=\right.$ $1,2, \ldots\}$ converge to a point $(t, y) \in U$. Then there exist a function $z \in Z^{-+}$, for which $t \in \pi(z), z(t)=y$, and a subsequence $\left\{z_{i_{j}} \mid j=1,2, \ldots\right\}$ such that for every segment I lying in $\pi(z)$ the inclusion $I \subseteq \pi\left(z_{i_{j}}\right)$ is satisfied beginning with some $j=j_{0}$ and the sequence $\left\{\left.z_{i_{j}}\right|_{I} \mid j=j_{0}, j_{0}+1, \ldots\right\}$ converges uniformly to the function $\left.z\right|_{I}$.

4. An equicontinuity condition and the position of the upper limit of a sequence of spaces. Keeping our suppositions about $U$, let us denote by $s(U)$ the set of all sequences $\left\{Z_{i} \mid i=1,2, \ldots\right\} \subseteq R^{i}(U)$ satisfying the condition

(4.1) for any $K \in \exp _{c} U$ each sequence $z_{j} \in\left(Z_{i_{j}}\right)_{K}, i_{1}<i_{2}<\ldots$, is equicontinuous.

Theorem 1.7 implies that every convergent sequence of elements of $R^{i}(U)$ belongs to the set $s(U)$. On the other hand, Theorem 1.8 implies

Proposition 4.1. Let $\alpha=\left\{Z_{i} \mid i=1,2, \ldots\right\} \in s(U)$ and $\lim \sup _{i \rightarrow \infty} Z_{i} \subseteq Z \subseteq$ $C_{s}(U)$. Then the sequence $\alpha$ converges in $U$ to the space $Z$.

Thus the proof of the convergence of a sequence $\alpha=\left\{Z_{i} \mid i=1,2, \ldots\right\} \subseteq R^{i}(U)$ to a space $Z \subseteq C_{s}(U)$ can be divided into two steps. The first one is the proof of the fulfillment of the condition $\alpha \in s(U)$. The second one is the proof of the fulfillment of the condition $\lim \sup _{i \rightarrow \infty} Z_{i} \subseteq Z$.

Our next aim is to point rather simple working conditions implying the fulfillment of the condition $\alpha \in s(U)$.

For $M \subseteq \mathbb{R} \times \mathbb{R}^{n}$ let $M_{t}=\left\{y \mid y \in \mathbb{R}^{n},(t, y) \in M\right\}$.

Let $\theta: U \rightarrow \mathbb{R}^{n}$ be a multifunction. For $(t, y) \in U$ let $\Gamma_{y}^{t}=\{\{y\} \cup H \mid H$ is an open subset of the set $U_{t}$ and $\left(O_{\varepsilon} y \cap \theta(t, y)\right) \backslash\{y\} \subseteq H$ for some $\left.\varepsilon>0\right\}$. Let us denote by $\tau(\theta, t)$ the topology on the set $U_{t}$ defined by the system of neighbourhoods $\left\{\Gamma_{y}^{t} \mid y \in U_{t}\right\}$.

THEOREM 4.1. Let $\alpha=\left\{Z_{i} \mid i=1,2, \ldots\right\} \subseteq R^{i}(U)$,

$$
\begin{aligned}
& \limsup _{\delta, \varepsilon \rightarrow 0, i \rightarrow \infty}\left\{\operatorname{Gr}\left(\left.z\right|_{\left[s_{1}, s_{2}\right]}\right) \mid z \in Z_{i},\left[s_{1}, s_{2}\right] \subseteq\right] t-\varepsilon, t+\varepsilon[\cap \pi(z), \\
&\left.z\left(\left[s_{1}, s_{2}\right]\right) \cap O_{\delta} y \neq \emptyset\right\} \subseteq \theta(t, y),
\end{aligned}
$$

$\gamma$ be a family of open subsets of the set $U,\left\{\left(Z_{i}\right)_{V} \mid i=1,2, \ldots\right\} \in s(V)$ for each $V \in \gamma$, for each $t \in \mathbb{R}$ the set $(U \backslash \cup \gamma)_{t}$ does not contain non-trivial connected subsets on which the topology $\tau(\theta, t)$ coincides with the Euclidean topology of $\mathbb{R}^{n}$. Then $\alpha \in s(U)$.

Note one trivial corollary of the theorem. The mapping $\theta \equiv \mathbb{R}^{n}$ satisfies all suppositions and so we get

Proposition 4.2. Let $\alpha=\left\{Z_{i} \mid i=1,2, \ldots\right\} \subseteq R^{i}(U), \gamma$ be a family of open subsets of the set $U,\left\{\left(Z_{i}\right)_{V} \mid i=1,2, \ldots\right\} \in s(V)$ for each $V \in \gamma$, for each $t \in \mathbb{R}$ the set $(U \backslash \cup \gamma)_{t}$ do not contain non-trivial connected subsets. Then $\alpha \in s(U)$.

Remark 4.1. Note one more situation where the fulfillment of condition (4.1) is obvious. Suppose that $\cup\left\{Z_{i} \mid i=1,2, \ldots\right\} \subseteq \Phi \in R_{c}^{i}(U)$. Then condition (4.1) holds. For example, let the right-hand sides of equations $y^{\prime}=f_{i}(t, y)$ satisfy the condition 
$\left\|f_{i}(t, y)\right\| \leq \varphi(t)$ with a Lebesgue integrable function $\varphi$. Thus all the solution spaces $D\left(f_{i}\right)$ lie in the solution space of the inequality $\left\|y^{\prime}(t)\right\| \leq \varphi(t)$. The last solution space satisfies condition (c), and this implies fulfillment of condition (4.1) for the sequence $\left\{D\left(f_{i}\right) \mid i=1,2, \ldots\right\}$.

Proposition 4.3. Let $X$ be a metric space, $\varphi: U \rightarrow X$ be a continuous mapping, $\alpha=\left\{Z_{i} \mid i=1,2, \ldots\right\} \subseteq R^{i}(U)$. Suppose that for every compact set $K \subseteq U$ and every sequence $z_{j} \in\left(Z_{i_{j}}\right)_{K}\left(i_{1}<i_{2}<\ldots\right)$ the sequence of functions

$$
\beta_{j}(t)=\varphi\left(t, z_{j}(t)\right), \quad j=1,2, \ldots, t \in \pi\left(z_{j}\right)
$$

is equicontinuous, $\gamma$ is a family of open subsets of the set $U$ and

$$
\left\{\left(Z_{i}\right)_{V} \mid i=1,2, \ldots\right\} \in s(V)
$$

for each $V \in \gamma$, for every $t \in \mathbb{R}$ and $x \in X$ the set

$$
(U \backslash \cup \gamma)_{t} \cap \varphi^{-1}(x)
$$

does not contain non-trivial connected subsets. Then $\alpha \in s(U)$.

Let $M \subseteq U$ and $\Psi \subseteq C_{s}(U)$. We shall say that the set $M \subseteq U$ is at most countable with respect to the space $\Psi$ and write $|M|_{\Psi} \leq \omega$, if $|\operatorname{Gr}(\psi) \cap M| \leq \omega$ for every function $\psi \in \Psi$ (here $\omega$ denotes the countable power).

TheOrem 4.2. Let $\alpha=\left\{Z_{i} \mid i=1,2, \ldots\right\} \subseteq R^{i}(U), \Phi=\lim \sup \alpha \subseteq \Psi \subseteq C_{s}(U)$, $Z \in R_{p}(U), \gamma$ be a family of open subsets of the set $U, \lim _{\sup _{i \rightarrow \infty}}\left(Z_{i}\right)_{V} \subseteq(Z)_{V}$ (in the space $\left.C_{s}(V)\right)$ for each $V \in \gamma$ and $|U \backslash \cup \gamma|_{\Psi} \leq \omega$. Then $\Phi \subseteq Z$.

The fact mentioned in Example 0.1 follows from Propositions 4.2 and 4.1 and Theorem 4.2. As we have mentioned in Section 3, such results can be applied to prove the fulfillment of condition (c), too. Stronger results on this topic and other working notions may be found in [4], [7]-[32]. Let us note some more statements of different levels about the convergence.

Proposition 4.4. Let $F: U \rightarrow \mathbb{R}^{n}$ be a multifunction, $z_{0} \in(D(F))^{-+}$, all limit points of the graph of the function $z_{0}$ at the end points of its domain lie outside $U$, $\alpha=\left\{Z_{i} \mid i=1,2, \ldots\right\} \subseteq R^{i}(U)$, the sequence $\left\{\left(Z_{i}\right)_{U \backslash \operatorname{Gr}\left(z_{0}\right)} \mid i=1,2, \ldots\right\}$ converge in $R\left(U \backslash \operatorname{Gr}\left(z_{0}\right)\right)$ to the space $D\left(F, U \backslash \operatorname{Gr}\left(z_{0}\right)\right)$. Then the sequence $\alpha$ converges to $D(F)$ in $R(U)$.

Applying the proposition to the stationary sequence $Z_{i} \equiv Z$ we obtain

Corollary. Let $F: U \rightarrow \mathbb{R}^{n}$ be a multifunction, $z_{0} \in(D(F))^{-+}$, all limit points of the graph of the function $z_{0}$ at the end points of its domain lie outside $U, D(F, U \backslash$ $\left.\operatorname{Gr}\left(z_{0}\right)\right) \in R_{c}\left(U \backslash \operatorname{Gr}\left(z_{0}\right)\right)$. Then $D(F) \in R_{c}(U)$.

Traditional results claim the continuity (for equations) and upper semicontinuity (for inclusions) of right-hand sides in some or all variables. The claiming is related in particular to a game with the following property of a solution space

(n) if a sequence $\left\{z_{i} \mid i=1,2, \ldots\right\} \subseteq Z$ of functions defined on a segment $[a, b] \subseteq$ $\mathbb{R}, a<b$, converges uniformly to a function $z \in Z$ then for a.e. $t \in[a, b]$ the 
derivatives $z^{\prime}(t), z_{i}^{\prime}(t), i=1,2, \ldots$, exist and $z^{\prime}(t) \in \cap\left\{\operatorname{cc}\left(\left\{z_{i}^{\prime}(t) \mid i \geq k\right\}\right) \mid i=\right.$ $1,2, \ldots\}$, where $\operatorname{cc}(M)$ denotes the closed convex envelope of a set $M$.

Remark 4.2. Basic situation where condition (n) is satisfied is the solution space of the inequality $\left\|y^{\prime}(t)\right\| \leq \varphi(t)$, where $\varphi$ is an arbitrary Lebesgue integrable function. The inequality is used in Carathéodory's and Davy's theorems). But this property is an example of "good" properties and the reasoning mentioned in section 2 suits to its investigation.

Basic results about the convergence of sequences of solution spaces look like the following two theorems.

THEOREM 4.3. Let

(4.2) $F_{k}: U \rightarrow \mathbb{R}^{n}$ for $k=0,1,2, \ldots, F: U \rightarrow \mathbb{R}^{n}$ be multifunctions,

(4.3) the space $D(F)$ satisfy conditions $(c)$ and $(n)$,

(4.4) $F_{k}(x) \subseteq F(x)$ for every $x \in U$ and $k=0,1,2, \ldots$,

(4.5) for every $\varepsilon>0$ there exists a set $E \subseteq U$, for which

a) $\mu(\{t \mid t \in \pi(\varphi),(t, \varphi(t)) \notin E\})<\varepsilon$ for every $\varphi \in D(F)$,

b) $E_{t}$ is closed in $U_{t}$ for every $t \in \mathbb{R}$,

c) $F_{0}(t, y) \supseteq \cap\left\{\operatorname{cc}\left(\cup\left\{F_{k}(\{t\} \times W) \mid k=0, i, i+1, i+2, \ldots\right\}\right), i=1,2, \ldots, W\right.$ is a neighborhood of $y$ in $\left.E_{t}\right\}$ for every $(t, y) \in E$.

Then the sequence $\left\{D\left(F_{k}\right) \mid k=1,2, \ldots\right\}$ converges to the space $D\left(F_{0}\right)$.

THEOREM 4.4. Let conditions $(4.2-4)$ hold,

(4.6) a set $V \subseteq U$ be open,

(4.7) $F_{0}(t, y) \supseteq \cap\left\{\operatorname{cc}\left(\cup\left\{F_{k}(\{t\} \times W) \mid k=0, i, i+1, i+2, \ldots\right\}\right), i=1,2, \ldots, W\right.$ is $a$ neighborhood of $y$ in $\left.U_{t}\right\}$ for every $(t, y) \in U \backslash V$.

(4.8) the sequence $\left\{D\left(F_{i}, V\right) \mid i=1,2, \ldots\right\}$ converge to the space $D\left(F_{0}, V\right)$.

Then the sequence $\left\{D\left(F_{k}\right) \mid k=1,2, \ldots\right\}$ converges to the space $D\left(F_{0}\right)$.

Remark 4.3. Remember our notes in the beginning of Section 3 about the position of the topological space $R_{c}(U)$ and the notion of the convergence of sequences of solution spaces. Thus we have pointed possibilities of proving propositions about continuity of the dependence of solutions on initial values and parameters in non-classical situations.

Remark 4.4. Compare the contents of this section with Theorems 3.1-3.3. We get the possibility of proving existence theorems, the fulfillment of conditions $(\mathrm{k})$ and $(\mathrm{h})$ in situations which are a long way from the classical theory. Let us show for instance how we can prove all these facts for the equation

$$
y^{\prime}=f(y)+g(t),
$$

where the function $f: \mathbb{R} \rightarrow[1, \infty[$ is measurable, the function $g: \mathbb{R} \rightarrow[0, \infty[$ is locally integrable. Let $Z_{f, g}$ denote the solution space of equation (4.9). Difficulties in the investigation of the equation are related to the joint discontinuity in the right-hand side both in $t$ and in $y$. 
Let $f_{i} \rightarrow f$ almost everywhere. Using propositions of the type mentioned above, we can prove the convergence

$$
Z_{f_{i}, g} \rightarrow Z_{f, g}
$$

Note that for the present we have no any existence theorem and we can not confirm that spaces under consideration have any non-trivial elements. But if "something" is contained in the spaces, "it" converges.

Applying to a stationary sequence $f_{i} \equiv f$ the previous observation about convergence (4.10) we get the belonging $Z_{f, g} \in R_{c}(\mathbb{R} \times \mathbb{R})$.

Next, every function $f$ of the mentioned type can be approximated by a sequence of continuous functions $\left\{f_{i} \mid i=1,2, \ldots\right\}$, in the sense that $f_{i}(t) \rightarrow f(t)$ as $i \rightarrow \infty$ for almost all $t$.

The equation

$$
y^{\prime}=f_{i}(y)+g(t)
$$

is covered by Carathéodory theorem, and thus $Z_{f_{i}, g} \in R_{c e}(\mathbb{R} \times \mathbb{R})$. Now Theorems 3.1-3.3 give us the existence theorem and the fulfillment of the conditions $(\mathrm{k})$ and $(\mathrm{h})$ for the equation (4.9).

Note one more situation where our considerations give a sufficient theory of the Cauchy problem.

THEOREM 4.5. Let the right-hand sides of the equations of the system

$$
\left\{\begin{array}{l}
x^{\prime}=f_{1}(x, y)+h_{1}(t, x, y) \\
y^{\prime}=f_{2}(x, y)+h_{2}(t, x, y)
\end{array}\right.
$$

be defined on an open subset $U \subseteq \mathbb{R} \times \mathbb{R}^{n}$, the functions $f_{1}$ (with values in $\mathbb{R}$ ) and $f_{2}$ (with values in $\left.\mathbb{R}^{n-1}\right)$ be bounded, continuous in $y$ and measurable in $x, f_{1}(x, y) \geq \varepsilon$ for a fixed $\varepsilon>0$ and every $(t, x, y) \in U$, the functions $h_{1}: U \rightarrow\left[0, \infty\left[\right.\right.$ and $h_{2}: U \rightarrow \mathbb{R}^{n-1}$ satisfy the Carathéodory's conditions. Then the solution space of the system satisfies conditions (c), (e) and (h).

5. Change of variables. Uniqueness of solutions. Consider the following situation being more general than the one which we have dealt with above.

Let $\pi: Y \rightarrow \mathbb{R}$ be a continuous mapping of a Hausdorff space $Y$ into the real line $\mathbb{R}$.

Denote by $\Sigma(Y, \pi)$ the set of all compact convex subsets $K$ of $Y$ satisfying the condition

$$
\text { the mapping }\left.\pi\right|_{K} \text { is injective. }
$$

It is well known that in this case the mapping $\left.\pi\right|_{K}: K \rightarrow \pi(K)$ is a homeomorphism and we can consider the continuous inverse mapping $f_{K}: \pi(K) \rightarrow K \subseteq Y$. The mapping $f_{K}$ is a partial selection for the multifunction $\pi^{-1}: \mathbb{R} \rightarrow Y$.

We will denote by $\pi\left(f_{K}\right)$ the set $K=\pi\left(\operatorname{Im} f_{K}\right)$ being the domain of the function $f_{K}$. So we shall denote the domain of the partial selection using the same symbol as the mapping under consideration.

Denote by $\Sigma_{s}(Y, \pi)$ the set of all such selections. The mapping Im associating to a function $f_{K} \in \Sigma_{s}(Y, \pi)$ its image $K \in \Sigma(Y, \pi)$ is bijective. 
The Vietoris topology is defined on the sets $\Sigma(Y, \pi) \subseteq \exp _{c} Y$. Define a topology on the set $\Sigma_{s}(Y, \pi)$ by the condition that the bijective mapping Im is an embedding.

This situation is obviously related to that one of Section 1. We do not repeat here topological remarks made there.

Let $Y \subseteq \mathbb{R} \times Y_{0}$ and $\pi$ denote the restriction to $Y$ of the projection $\mathbb{R} \times Y_{0} \rightarrow \mathbb{R}$. Let $p: \mathbb{R} \times Y_{0} \rightarrow Y_{0}$ be the second projection. In this case we can associate to each function $f \in \Sigma_{s}(Y, \pi)$ the function $p f: \pi(f) \rightarrow Y_{0}$. Such function $p f$ is a partial mapping of $\mathbb{R}$ into $Y_{0}$ and we get the situation considered above. The mapping $P: \Sigma_{s}(Y, \pi) \rightarrow C_{s}(Y)$, where $P(z)=p z$, is a homeomorphism.

The statements about $C_{s}(Y), R^{i}(Y), R(Y)$, etc. have its obvious versions for $\Sigma_{s}(Y, \pi)$ and related structures. But we have mentioned these notions only to point out that this level is natural for a category corresponding to our considerations. Let us introduce morphisms of the structures in question.

Let $\pi: Y \rightarrow \mathbb{R}, \pi_{1}: Y_{1} \rightarrow \mathbb{R}$ and $\Phi: Y \rightarrow Y_{1}$ be continuous mappings.

We shall say that the mapping $\Phi$ is extendable to a space $Z \subseteq \Sigma_{s}(Y, \pi)$ if for each function $z \in Z$ the set $\Phi(\operatorname{Im}(z))$ belongs to $\Sigma_{s}\left(Y_{1}, \pi_{1}\right)$ (we shall denote it by $\widetilde{\Phi}(z)$ ) and either

$(+)$ the function $\pi_{1}(\Phi(t, z(t)))$ is increasing for each $z \in Z$,

or

(-) the function $\pi_{1}(\Phi(t, z(t)))$ is decreasing for each $z \in Z$.

A homeomorphism $\Phi: Y \rightarrow Y_{1}$ satisfying this condition will be called a change of variables for the space $Z \subseteq \Sigma_{s}(Y, \pi)$.

Under the assumptions of Section 2 so defined change of variables keeps the fulfillment of conditions 1, 2, (e), (c) and (u) of Section 2. Condition $(\mathrm{k})$ is keeping under assumption of the fulfillment of conditions (c) and (e). Below we extend the notation $\widetilde{\Phi}(z)$ to elements of $C_{s}(U)$.

Some particular changes of variables will be important for us.

Let $\varphi_{h \lambda}(t, y)=\left(t-h, \lambda^{-1} y\right)$ for $h \geq 0, \lambda>0$ and $(t, y) \in \mathbb{R} \times \mathbb{R}^{n}$. Let $\Psi_{\tau}(t, y)=$ $(t-\tau, y)$ for $\tau \in \mathbb{R}$ and $(t, y) \in \mathbb{R} \times \mathbb{R}^{n}\left(\Psi_{\tau} \equiv \varphi_{\tau 1}\right.$ and $\left.\varphi_{\tau \lambda} \equiv \Psi_{\tau} \varphi_{0 \lambda} \equiv \varphi_{0 \lambda} \Psi_{\tau}\right)$. The mappings $\varphi_{h \lambda}$ and $\Psi_{\tau}$ are changes of variables for every space $Y \subseteq C_{s}\left(\mathbb{R} \times \mathbb{R}^{n}\right)$.

The change of variables $\varphi_{0 \lambda}$ transfers the space of solutions of an equation $y^{\prime}=f(t, y)$ to the space of solutions of the equation $y^{\prime}=\lambda^{-1} f(t, \lambda y)$. The change of variables $\Psi_{\tau}$ transfers the space of solutions of an equation $y^{\prime}=f(t, y)$ to the space of solutions of the equation $y^{\prime}=f(t+\tau, y)$

Returning to a general case denote by $G R^{i}(Y, \pi)$ the set of all spaces $Z \subseteq \Sigma_{s}(Y, \pi)$ satisfying condition (1) of section 2 .

If a continuous mapping $\Phi: Y \rightarrow Y_{1}$ is extendable to a space $Z \subseteq \Sigma_{s}(Y, \pi)$, we have a continuous mapping $\widetilde{\Phi}: Z \rightarrow \Sigma_{s}\left(Y_{1}, \pi_{1}\right)$ and

$$
\text { if } Z \in G R^{i}(Y, \pi) \text {, then } \widetilde{\Phi}(Z) \in G R^{i}\left(Y_{1}, \pi_{1}\right) \text {. }
$$

This is one of the most important properties of the class $G R^{i}(Y, \pi)$ (and, of course, of the class $\left.R^{i}(U)\right)$. Its other important properties which determine its central position in such questions are: 
if $\zeta \subseteq G R^{i}(Y, \pi)$, then $\cup \zeta \in G R^{i}(Y, \pi)$,

if $\zeta \subseteq G R_{c}^{i}(Y, \pi)$, then $\cup \zeta \in G R_{c}^{i}(Y, \pi)$,

if the mapping $\Phi$ is perfect and $Z \in G R_{c}^{i}(Y, \pi)$, then $\widetilde{\Phi}(Z) \in G R_{c}^{i}\left(Y_{1}, \pi_{1}\right)$.

Consider now the problem of the uniqueness of solutions. Let

(5.2) $X, Y_{0}$ be locally compact metric spaces, $Y=\mathbb{R} \times Y_{0}$,

(5.3) $y_{0} \in Y_{0}, \pi_{1}: Y \rightarrow \mathbb{R}$ be the projection, $\pi: X \rightarrow \mathbb{R}$ be a continuous mapping,

(5.4) $Z \in G R^{i}(X, \pi), z_{0} \in Z, 0 \in \pi\left(z_{0}\right)$,

(5.5) $\left\{U_{j} \mid j=1,2, \ldots\right\}$ be a base of neighborhoods of the set $\operatorname{Im}\left(z_{0}\right)$ in the subspace $X \cap \pi^{-1}\left(z_{0}\right)$, let continuous mappings $\psi_{j}:\left[U_{j}\right] \rightarrow Y, j=1,2, \ldots$, be extendable to the space $Z$, and let

$(*) \operatorname{Im}\left(z_{0}\right) \subseteq \psi_{j}^{-1}\left(\mathbb{R} \times\left\{y_{0}\right\}\right)$,

(**) for every neighborhood $O y_{0}$ of the point $y_{0}$ in the space $Y$, beginning with some $j, \psi_{j}\left(\partial U_{j}\right) \subseteq \mathbb{R} \times\left(Y_{0} \backslash O y_{0}\right)$,

$(* * *)$ if $\pi\left(x_{1}\right) \leq 0=\pi\left(x_{2}\right) \leq \pi\left(x_{3}\right)$, then $\pi\left(x_{1}\right) \leq \pi_{1}\left(\psi_{j}\left(x_{1}\right)\right) \leq 0=\pi_{1}\left(\psi_{j}\left(x_{2}\right)\right) \leq$ $\pi_{1}\left(\psi_{j}\left(x_{3}\right)\right) \leq \pi\left(x_{3}\right)$

(5.6) $\gamma$ be a family of open subsets $V$ of the space $Y$ which does not contain the point $\left(0, y_{0}\right)$ and satisfies the condition

(*) for each compact set $K \subseteq V$ every sequence $z_{j} \in\left(\widetilde{\psi}_{j}(Z)\right)_{K}, j=1,2, \ldots$ is equicontinuous,

(5.7) a set $H \subseteq Y$ contains the set

$\limsup _{\varepsilon \rightarrow 0, j \rightarrow \infty}\left\{\operatorname{Im}\left(\left.z\right|_{\left[s_{1}, s_{2}\right]}\right) \mid z \in \widetilde{\psi}_{j}(Z),\left[s_{1}, s_{2}\right] \subseteq\right]-\varepsilon, \varepsilon\left[\cap \pi(z),\left(0, y_{0}\right) \in z\left(\left[s_{1}, s_{2}\right]\right)\right\}$.

THEOREM 5.1. Let conditions (5.2)-(5.7) hold and the component of the point $\left(0, y_{0}\right)$ in the set $H \backslash \cup \gamma$ be trivial, $z \in Z, 0 \in \pi(z)$, and $z(0)=z_{0}(0)$. Then $\left.z\right|_{[-\varepsilon, \varepsilon] \cap \pi(z) \cap \pi\left(z_{0}\right)}=$ $\left.z_{0}\right|_{[-\varepsilon, \varepsilon] \cap \pi(z) \cap \pi\left(z_{0}\right)}$ for some $\varepsilon>0$.

Theorem 5.1 is ready for applications in the investigation of the uniqueness of solutions but the situation is described there in words which are unusual for the TODE. So note its corollaries where the description of the situation is closer to applications.

TheOrem 5.2. Let $z \in Z \in R^{i}(U), 0 \in \pi(z), z(0)=\overline{0}$, conditions (5.5)-(5.6) hold, the component of the point $\left(0, y_{0}\right)$ in the set $\left(\{0\} \times \mathbb{R}^{k}\right) \backslash \cup \gamma$ be trivial. Then $\left.z\right|_{[-\varepsilon, \varepsilon] \cap \pi(z)} \equiv \overline{0}$ for some $\varepsilon>0$.

Theorem 5.3. Let $z \in Z \in R_{c}(U), 0 \in \pi(z), z(0)=\overline{0}, \psi_{j}=\varphi_{0 \lambda_{j}}$ for some sequence $\lambda_{j} \rightarrow 0$, the sequence of spaces $\left\{\widetilde{\psi}_{j}(Z) \mid j=1,2, \ldots\right\}$ have a limit in the space $R_{c}(U)$. Then $\left.z\right|_{[-\varepsilon, \varepsilon] \cap \pi(z)} \equiv \overline{0}$ for some $\varepsilon>0$.

Remark 5.1. The classical uniqueness theorem with the Lipschitz condition is a trivial corollary of our last theorem.

ExAmple 5.1. Consider the scalar equation $y^{\prime}=y|t|^{\sin (\ln |y|)}(=0$ for $y=0)$. Aiming to prove the uniqueness of the solution $z=0$ for the Cauchy problem $y\left(t_{0}\right)=0$, let us consider the mappings $\psi_{i}(t, y)=(t, y \exp (2 \pi i)), i=1,2, \ldots$. This changes of variables keep the solution space of the equation in question. 
6. General concepts. The basic properties of solution spaces noted above may be taken as axioms for some geometric chapters of the TODE. This allows us to introduce most general concepts of some notions related to properties of equations and their solutions and use them in real investigation of equations. The tools prepared in previous sections make this approach working in situations not covered by the classical theory.

Let $V$ be an open subset of the space $\mathbb{R}^{n}$. A space $Z \subseteq C_{s}(\mathbb{R} \times V)$ will be called autonomous if $\Psi_{\tau}(Z)=Z$ for every $\tau \in \mathbb{R}$.

It is well known that the space of solutions of an autonomous equation $y^{\prime}=f(y)$ is autonomous.

The set of all autonomous spaces $Z \in R(\mathbb{R} \times V)$ will be denoted by $A(V)$. Let $*$ be an arbitrary set of conditions from our list of conditions on elements of $R(U)$ in Section 2 . Let $A_{*}(V)=A(V) \cap R_{*}(\mathbb{R} \times V)$.

The study of geometrical properties of solutions of autonomous equations (systems) with continuous right-hand sides is often simplified by the fact that in a neighborhood of a non-stationary point the moving along the trajectories is rather directed. Under our assumptions we have no such strong directness but there are still possibilities of weaker estimates.

For $Z \in A(V)$ and $M \subseteq V$ let $\operatorname{diam}_{Z} M=\sup \{b-a \mid[a, b]=\pi(z), z \in Z, \operatorname{Im}(z) \subseteq$ $M\}$ (the diameter of the set $M$ with respect to the space $Z$ ).

Proposition 6.1. Let $Z \in A_{c e}(V), K \in \exp _{c}(V)$ and $\operatorname{diam}_{Z} K=\infty$. Then there exists a function $z \in Z^{+}$for which $\operatorname{Im}(z) \subseteq K$ and $\pi(z)=[0, \infty[$.

Proposition 6.2. Let $Z \in A_{c}(V),\left\{K_{i} \mid i=1,2, \ldots\right\} \subseteq \exp _{c}(V), K_{1} \supseteq K_{2} \supseteq K_{3} \supseteq$ $\ldots, a \geq 0$ and $\operatorname{diam}_{Z} K_{i} \geq a$ for every $i=1,2, \ldots$ Then $\operatorname{diam}_{Z} \cap\left\{K_{i} \mid i=1,2, \ldots\right\} \geq a$.

A point $y \in V$ will be called a stationary point of a space $Z \in A(V)$ if the function $z \equiv y$ defined on $\mathbb{R}$ belongs to $Z^{-+}$.

It is clear that the diameter of a stationary point equals $\infty$, the diameter of a nonstationary one equals 0. It follows from Proposition 6.2 that for every stationary point $y \in V$ and $\varepsilon>0$ there exists a neighborhood $O y$ having diameter less than $\varepsilon$. In a neighborhood of small diameter the moving along trajectories is directed not so strictly as in the case of an equation with continuous right-hand side but sufficiently for some observations.

Let $G$ be an open subset of the plane $\mathbb{R}^{2}$.

THEOREM 6.1. Let $z \in Z \in A_{c e}(G), \pi(z)=[a, b], a<b, z(a)=z(b)$ and $z\left(t_{1}\right) \neq z\left(t_{2}\right)$ for $a \leq t_{1}<t_{2}<b$, the domain $H \subseteq G$ is bounded by the curve $z(t), t \in \pi(z)$. Then there exists a stationary point $y \in[G]$ of the space $Z$.

Let $Z \in A_{c e k}(G)$.

Let $x \in G, \varepsilon>0, K=\left[O_{\varepsilon} x\right] \subseteq G, \operatorname{diam}_{Z} K<\infty$. Let $S$ be a circle bounding the disk $K$.

Let $M=\{z \mid z \in Z, 0 \in \pi(z), z(0)=x, \operatorname{Im}(z) \subseteq K, \alpha(z), \beta(z) \in S\}$, where $\alpha(z)=z(\inf \pi(z)), \beta(z)=z(\sup \pi(z))$. Our assumptions imply that $\alpha(M) \cap \beta(M)=\emptyset$. Fix any function $z_{0} \in M$ and denote by $\gamma_{1}$ (resp. by $\gamma_{2}$ ) the set of all segments of the circle $S$ with ends from $\alpha(M)$ (resp. $\beta(M)$ ), containing the point $\alpha\left(z_{0}\right)$ (resp. $\beta\left(z_{0}\right)$ ). 
Segments $p_{i}=\cup \gamma_{i}(i=1,2)$ are defined independently of our choice of the function $z_{0}$. Let $P(x, \varepsilon)=\left\{(u-x) \varepsilon^{-1} \mid u \in p_{2}\right\}$ and $G_{P}=\left\{(x, \delta) \mid x \in G, \delta>0, \operatorname{diam}_{Z} O_{\delta} x<\infty\right\}$. So $P$ is a multivalued mapping from $G_{P}$ to the circle $S_{1}=\left\{u \mid u \in \mathbb{R}^{2},\|u\|=1\right\}$ with proper connected compact subsets of $S_{1}$ as values. The degree theory is well known for such mappings. So we get the possibility to develop the index theory for the space $Z \in A_{c e k}(G)$.

One of real meanings of this possibility is related with situation when we can calculate the index using a way lying in a domain without singularities. So classical propositions suit to do it. But the result of the calculation is applicable to the equation under consideration which may have singularities.

Let now $V$ be an open subset of the plane $\left.\mathbb{R}^{2}, a \in \mathbb{R} \cup\{-\infty\}, U=\right] a, \infty[\times V$.

We shall say that a space $X$ converges to a space $Z \in R(U)$ as $t \rightarrow \infty$ if for every sequence $\delta_{i} \rightarrow \infty$ the sequence of the spaces $\left\{\widetilde{\Psi}_{\delta_{i}}(X) \mid i=1,2, \ldots\right\}$ has a subsequence converging to the space $Z$. .

Theorem 6.2. Let a space $X \in R(U)$ converge to a space $Z \in A_{c e}(V)$ as $t \rightarrow \infty$, $z \in Z^{+}, \pi(z)=\left[b, \infty\left[, z(s) \neq z(t)\right.\right.$ for $s>t>b$. Suppose that the set $\Omega^{+}(z) \subseteq V$ is non-empty and compact and does not contain stationary points of the space $Z_{\infty}$. Then there exists a function $z_{0} \in \cup Z_{\infty}$ for which $\Omega^{+}(z)=\operatorname{Im}\left(z_{0}\right), z_{0}\left(\inf \pi\left(z_{0}\right)\right)=z_{0}\left(\sup \pi\left(z_{0}\right)\right)$ and $z_{0}(s) \neq z_{0}(t)$ for $\inf \pi\left(z_{0}\right) \leq s<t<\sup \pi\left(z_{0}\right)$.

In the case $Z_{\infty}=Z$ Theorem 6.2 gives us a version of the Poincaré-Bendixson theorem (see Theorem VII.4.1 in [33]) which is applicable to equations with discontinuous righthand sides. See also [35] and [41].

Another situation when we can propose a new very general notion is investigation of stationary points "in first approximation". Common approach uses the language related to $o$-small terms. Those are estimates in the metric of uniform convergence. Our topological structure offers us a possibility of using stronger tools in description of the corresponding situations.

The general idea here is easy to explain. When we want to study the behavior of solutions of the equation $y^{\prime}=f(t, y)$ near the stationary point $y=\overline{0}$ we consider the changes of variables $\varphi_{0 \lambda}$ (see Section 5) and we prove then that the solution spaces of equations obtained under this change of variables $\left(y^{\prime}=\lambda f(t, y / \lambda)\right)$ converge to the solution space of an equation $y^{\prime}=g(t, y)$ as $\lambda \rightarrow 0$. In this case asymptotic properties of solutions of the equation $y^{\prime}=f(t, y)$ near the point $y=\overline{0}$ are similar to the properties of solutions of the equation $y^{\prime}=g(t, y)$. If we prove the mentioned convergence using classical theorems on the continuity of the dependence of solutions on parameters of the equations, we obtain the classical theory of "first approximation" asymptotic integration. Our new methods give us a possibility to investigate various other situations.

Following this approach we can consider the system

$$
\left\{\begin{array}{l}
x^{\prime}=-2 x-y \\
y^{\prime}=x-2 y
\end{array}\right.
$$


as the "first approximation" of the system

$$
\left\{\begin{array}{l}
x^{\prime}=-2 x-y \\
y^{\prime}=x-2 y+x^{-1} e^{-|y| / x^{2}}
\end{array}\right.
$$

although the right-hand side of the second equation is far away from usual assumptions of the "first approximation". If $y=0$ and $x \rightarrow 0$, the additional term tends to $\infty$. We can prove the stability and obtain estimates of asymptotic integration in such situations.

The same ideas may be used to estimate the behavior of solutions at infinity. As in the generalization of the "first approximation" we consider changes of variables $(t, y) \rightarrow$ $(t, \lambda y)$ but now we prove the corresponding convergence or make other observation as $\lambda \rightarrow 0$. In particular, we have

Theorem 6.3. Let $\left.r \geq 0, V_{r}=\left\{u \mid u \in \mathbb{R}^{n},\|u\|>r\right\}, U_{r}=\right] a, b\left[\times V_{r}, Z \in R^{i}\left(U_{r}\right)\right.$, $\lambda_{1}>\lambda_{2}>\ldots, \lambda_{i} \rightarrow 0$ as $i \rightarrow \infty, \psi_{i}(t, y)=\left(t, \lambda_{i} y\right), \gamma$ be a family of open subsets of the set $U$ and

$$
\left\{\left(\widetilde{\psi}_{i}\left(Z_{i}\right)\right)_{V} \mid i=1,2, \ldots\right\} \in s(V)
$$

for each $\left.V \in \gamma,\left[t_{0}, t_{1}\right] \subseteq\right] a, b\left[\right.$ and for every point $t \in\left[t_{0}, t_{1}\right]$ all components of the set $\left(\{t\} \times V_{r}\right) \backslash \cup \gamma$ be bounded. Then every function from the set $M=\left\{z \mid z \in Z^{-} \cup\right.$ $\left.Z^{+}, \pi(z) \subseteq\left[t_{0}, t_{1}\right]\right\}$ is bounded.

The meaning of this theorem is a possibility to estimate the length of the interval of existence of a solution. We get

Corollary. Let $\left.r \geq 0, V_{r}=\left\{u \mid u \in \mathbb{R}^{n},\|u\|>r\right\}, U_{r}=\right] a, b\left[\times V_{r}, U=\right] a, b\left[\times \mathbb{R}^{n}\right.$, $Z \in R^{i}(U), Z_{\infty} \in R^{i}\left(U_{r}\right), \lambda_{1}>\lambda_{2}>\ldots, \lambda_{i} \rightarrow 0$ as $i \rightarrow \infty, \psi_{i}(t, y)=\left(t, \lambda_{i} y\right)$, $\left(\widetilde{\psi}_{i}(Z)\right)_{U_{r}} \rightarrow Z_{\infty}$. If the domain of definition $\pi(z)$ of a function $z \in Z^{-+}$intersects the segment $\left[t_{0}, t_{1}\right]$, then $\left[t_{0}, t_{1}\right] \subseteq \pi(z)$.

7. On homological properties of solution spaces. Applications of the LeraySchauder theory in the theory of ordinary differential equations are based on estimates of homological properties of the corresponding integral operators. But if the right-hand side of the equation under consideration is discontinuous, the integral operator need not exist. Our possibility of description of topological properties at the level of solution spaces allows us to construct an equivalent of the Leray-Schauder theory which can be applied to equations with discontinuous right-hand sides.

Note first that some notions introduced before keep their value for our topological structures. In particular, the theory from Sections 1-6 of [8] can be rather easily translated to the level of topology of the space of solution spaces, and its enriched version can be applied to investigation of equations and inclusions with right-hand sides with complicated discontinuities. For instance, in this way we can obtain the following

TheOREM 7.1. Let $U=\mathbb{R} \times \mathbb{R}^{n}, \omega>0, A$ be a constant matrix of the order $n$ and the equation $y^{\prime}=A y$ have no nontrivial $\omega$-periodic solutions, $Z$ be the solution space of the equation $y^{\prime}=A y$, a space $Y \in R_{c e h}(U)$ be $\omega$-periodic, $\lambda_{1}>\lambda_{2}>\ldots, \lambda_{i} \rightarrow 0$, $\psi_{i}(t, y)=\left(t, \lambda_{i} y\right)$ and $\widetilde{\psi}_{i}(Y) \rightarrow Z$ as $i \rightarrow \infty$. Then there exists a $y \in Y$ such that $\pi(y)=[0,1]$ and $y(0)=y(\omega)$. 
ExAmple 7.1. Theorem 7.1 can be applied for instance in the following situation. Let $A$ be a constant matrix of the order $n$ and the equation $y^{\prime}=A y$ have no nontrivial $\omega$-periodic solutions, a function $f: \mathbb{R} \times \mathbb{R}^{n} \rightarrow \mathbb{R}^{n}$ be measurable and $\omega$-periodic in the first argument (in "the time" $t \in \mathbb{R}$ ), be continuous in the second argument (in "the space variable" $\left.y \in \mathbb{R}^{n}\right), \varphi(t)$ be non negative Lebesgue locally integrable function and $\|f(t, y)\| \leq \varphi(t)$ for every $t \in \mathbb{R}$ and $y \in \mathbb{R}^{n}$. Let $u \in \mathbb{R}^{n}, 0 \leq \alpha<1$ and for $y=\left(y_{1}, \ldots, y_{n}\right)$

$$
g(t, y)=\|y\|^{\alpha}|\sin t|^{\sin \left(\ln \left|y_{1}\right|\right)+1-\frac{\left|y_{1}\right|}{\|y\|},}
$$

$(g(t, y)=0$ in all cases where the previous formula does not define the corresponding value). Using Theorem 7.1 with $\lambda_{i}=2 \pi i$ we can prove that the equation

$$
y^{\prime}=A y+f(t, y)+u g(t, y)
$$

has a $2 \pi$-periodic solution (attract the reader's attention to singularities of the function $g$ when $\left(\frac{3 \pi}{2}+2 \pi i, 0, \ldots, 0\right)$ and $\left.\sin t \rightarrow 0\right)$.

Now we pass to remarks about an equivalent of the Leray-Schauder theory. Here we will not give a large account of a perfect theory and we will point only a possibility to create it. We start with the Dirichlet problem. Let

$$
\begin{aligned}
& U=] a, b\left[\times \mathbb{R}^{2}, \quad \text { where }-\infty<a<b<\infty,\right. \\
& Z \in R_{c e}(U)
\end{aligned}
$$

(below we will use stronger conditions). The boundary conditions for the Dirichlet problem we will write as

$$
x(0)=x(1)=0, \quad \text { where } z=(x, y) \in Z \text { and } \pi(z)=[0,1] .
$$

Applications of the Leray-Schauder theory use homotopies and a priori estimates. We will start from the same positions. Let

(7.4) $-\infty<c<d<\infty, Z_{\lambda} \in R_{c e k}(U)$ for every $\lambda \in[c, d]$ and the mapping $\varphi:[c, d] \rightarrow$ $R_{c e k}(U)$, defined by the formula $\varphi(\lambda)=Z_{\lambda}$, be continuous,

(we introduce here a homotopy in the same way as in applications of the Leray-Schauder theory. In classical situations the continuity of the mapping $\varphi$ follows from standard theorems on the continuity of the dependence of solutions on parameters in right-hand side. In more complicated situation we can apply tools of our approach),

(7.5) $-\infty<B<C<\infty$ and for every $\lambda \in[c, d]$ each solution of problem for the space $Z=Z_{\lambda}$ satisfies the condition $y(0), y(1) \in[B, C]$.

We shall formulate three results in this direction.

THEOREM 7.2. Let conditions (7.1), (7.4) and (7.5) hold,

(7.7) for every $\lambda \in[c, d]$ every solution of the Cauchy problem

$$
z=(x, y) \in Z_{\lambda}^{+}, \quad x(0)=0, \quad y(0) \in[A, D]
$$

satisfies the condition $[0,1] \subseteq \pi(z)$, 
(7.8) the axis of ordinates separates the sets $\left\{z(1) \mid z \in Z_{c}^{+}, z(0)=(0, A)\right\}$ and $\{z(1) \mid$ $\left.z \in Z_{c}^{+}, z(0)=(0, D)\right\}$.

Then

(7.9) there exists a function $z=(x, y) \in Z_{d}$ for which $x(0)=x(1)=0$.

THEOREM 7.3. Let condition (7.1) hold,

(7.10) $-\infty<c<d<\infty, Z_{\lambda} \in R_{\text {ceu }}(U)$ for every $\lambda \in[c, d]$, and the mapping $\varphi:[c, d] \rightarrow$ $R_{\text {ceu }}(U)$ defined by the formula $\varphi(\lambda)=Z_{\lambda}$, be continuous,

conditions (7.5), (7.6), (7.8) hold and

(7.11) every solution of the Cauchy problem

$$
z=(x, y) \in Z_{c}^{+}, x(0)=0, y(0) \in[A, D]
$$

satisfies the condition $[0,1] \subseteq \pi(z)$,

(7.12) there exists a number $R>0$, such that for every $\lambda \in[c, d]$ every solution of the Dirichlet problem

satisfies the condition $\|z\|<R$.

$$
z=(x, y) \in Z_{\lambda}^{+}, \quad x(0)=x(1)=0,
$$

Then condition (7.9) is satisfied.

We proceed now to the main results of this section. Theorem 7.3 is an easy particular case of the following

TheOREM 7.4. Let condition (1) of Section 2 hold,

(7.13) $-\infty<c<d<\infty, Z_{\lambda} \in R_{\text {ceh }}(U)$ for every $\lambda \in[c, d]$ and the mapping $\varphi$ : $[c, d] \rightarrow R_{c e h}(U)$, defined by formula $\varphi(\lambda)=Z_{\lambda}$ be continuous, conditions (7.5), (7.6), (7.8), (7.11) and (7.12) hold.

Then condition (7.9) is satisfied.

Now we shall consider the problem of existence of periodic solutions. Let

$$
\begin{aligned}
& U=\mathbb{R} \times \mathbb{R}^{n}, \\
& Z \in R_{\text {ceh }}(U) .
\end{aligned}
$$

We can understand the problem of the existence of a periodic solution as the problem of the existence of a function

$$
z \in Z
$$

such that

$$
\pi(z)=[0,1] \quad \text { and } \quad z(0)=z(1)
$$

Let also

$$
r>0, \quad S=\left\{u \mid u \in \mathbb{R}^{n},\|u\|=r\right\}, \quad K=\left\{u \mid u \in \mathbb{R}^{n},\|u\| \leq r\right\},
$$

(7.19) every function $z \in Z^{+}$, for which $\inf \pi(z)=0$ and $z(0) \in K$, satisfies the condition $\pi(z) \supseteq[0,1]$, 
(7.20) every function $z \in Z^{+}$, for which $\inf \pi(z)=0$ and $z(0) \in S$, satisfies the condition $z(0) \neq z(1)$.

It is easy to recognize in the last condition a version of an a priori estimate usually used in applications of the Leray-Schauder method.

Under hypotheses (7.14)-(7.15), (7.18)-(7.20) we can define in a standard manner an index of the solution space $Z$ in the segment $[0,1]$ with respect to the sphere $S$. We shall denote it by $k(Z, S)$. If $k(Z, S) \neq 0$, problem (7.16)-(7.17) has a solution (here the situation is completely analogous to the one of $\S 6$ in $[8])$.

Theorem 7.5. Let conditions (7.14), (7.18), (7.13) hold,

(7.21) the space $Z=Z_{c}$ satisfy conditions (7.19)-(7.20) and $k(Z, S) \neq 0$,

(7.22) for every $\lambda \in[c, d]$ every solution of the problem $z \in Z_{\lambda}$, (7.17) satisfy the condition $z(0) \in K \backslash S$.

Then the problem $z \in Z_{d}$, (7.17) has a solution.

Theorem 7.6. Let conditions (7.14), (7.18), (7.13), (7.21) hold,

(7.23) every solution of the problem $z \in Z_{c}$, (7.17) satisfy the condition $z(0) \in K \backslash S$,

(7.24) the set $\Sigma=\left\{(\lambda, z) \mid \lambda \in[c, d], z \in Z_{\lambda}, \pi(z)=[0,1], z(0)=z(1)\right\} \subseteq[c, d] \times$ $C\left([0,1], \mathbb{R}^{n}\right)$ can be represented as the union of a discrete family of compacts.

Then the problem $z \in Z_{d}$, (7.17) has a solution.

A natural first impression of some artificiality of condition (7.24) disappears when we observe that if periodic trajectories of an equation under consideration are related to revolving around any fixed set, the function $s(z)$, defined as the number of rotations accomplished by the periodic solution $z$, is locally constant. When we have corresponding a priori estimates, this gives the needed representation.

\section{References}

[1] R. Bielawski, L. Górniewicz and S. Plaskacz, Topological approach to differential inclusions on closed subsets of $\mathbb{R}^{n}$, Dynamics reported, N1 (new series), Springer-Verlag, 1992, 225-250.

[2] E. A. Coddington and N. Levinson, Theory of ordinary differential equations, McGraw Hill, New York, 1955.

[3] J. L. Davy, Properties of solution set of generalized differential equation, Bull. Austral. Math. Soc. 6 No3 (1972), 379-398.

[4] V. V. Fedorchuk and V. V. Filippov, General topology, Basic constructions. Moscow University Publishers, 1988.

[5] A. F. Filippov, Differential equations with discontinuous right-hand side, Nauka, 1985.

[6] A. F. Filippov, Differential equations with discontinuous right-hand side, Matem. Sbornik, 51 N1 (1960), 99-128.

[7] V. V. Filippov, Topological structure of solution spaces of ordinary differential equations, Uspekhi Mat. Nauk 48 N1 (1993), 103-154. 
[8] V. V. Filippov, Solution spaces of ordinary differential equations, Moscow University Publishers, 1993.

[9] V. V. Filippov, On the existence and properties of solutions of ordinary differential equations and differential inclusions, Doklady AN SSSR, 279 N1 (1984), 47-50.

[10] V. V. Filippov, Axiomatic theory of solution spaces of ordinary differential equations, Doklady AN SSSR, 280 N2 (1985), 304-308.

[11] V. V. Filippov, On the theory of solution spaces of ordinary differential equations, Doklady AN SSSR, 285 N5 (1985), 1073-1077.

[12] V. V. Filippov, On ordinary differential equations with singularities in right-hand side, Mat. zametki 38 N6 (1985), 832-851.

[13] V. V. Filippov, On the Luzin theorem and right-hand sides of differential inclusions, Mat. zametki 37 N1 (1985), 93-98.

[14] V. V. Filippov, On the existence and properties of solutions of ordinary differential equations, Differen. Uravnen. 22 N6 (1986), 968-977.

[15] V. V. Filippov, On the theorem of existence of solutions of ordinary differential equations, Mat. Zametki 40 N6 (1986), 743-748.

[16] V. V. Filippov, On the theorems of Luzin and Scorza-Dragoni, Vestnik Moskov. Univers. Ser.1, Math.,Mech., N3 (1987), 66-68.

[17] V. V. Filippov, On the Kneser theorem, Differen. Uravnen. 23 N12 (1987), 2068-2074.

[18] V. V. Filippov, A note on the Kneser theorem, Vestnik Moskov. Univers. Ser.1, Math. Mech. N1 (1987), 61-63.

[19] V. V. Filippov, Two notes on the theory of solution spaces of ordinary differential equations, Vestnik Moskov. Univers. Ser.1, Math. Mech. N4 (1988), 53-55.

[20] V. V. Filippov, The theory of the Cauchy problem for ordinary differential equations from the point of view of general topology, in: General topology. Mappings of topological spaces, Moscow University Publishers, 1988.

[21] V. V. Filippov, On the asymptotic integration of ordinary differential equations with discontinuity in the right-hand side, Doklady AN SSSR, 321 N3 (1991), 482-485.

[22] V. V. Filippov, On stationary points and some geometric properties of solutions of ordinary differential equations, Doklady AN SSSR, 323 N6 (1992), 1043-1047.

[23] V. V. Filippov, On the investigation of ordinary differential equations "in first approximation", Differen. Uravnen. 28 N8 (1992), 1351-1355.

[24] V. V. Filippov, On the asymptotics of solutions of quasilinear ordinary differential equations, Differen. Uravnen. 28 N10 (1992), 1747-1751.

[25] V. V. Filippov, On the Poincaré-Bendixson theorem and compact families of solution spaces of ordinary differential equations, Mat. Zametki 53 N2 (1993), 140-144.

[26] V. V. Filippov, On families of changes of variables and the uniqueness theorem for ordinary differential equations, Doklady RAN, 330 N6 (1993), 704-706.

[27] V. V. Filippov, A note on one theorem of I. T. Kiguradze, Vestnik Moskov. Univers. Ser.1, Math. Mech. N4 (1993), 11-13.

[28] V. V. Filippov, On the investigation of stationary points in the plane, Vestnik Moskov. Univers. Ser.1, Math. Mech. N5 (1993), 3-10.

[29] V. V. Filippov, On the theory of the Cauchy problem for an ordinary differential equation with discontinuous right-hand side, Matem. Sbornik N11 (1994), 95-118.

[30] V. V. Filippov, On sequences of changes of variables and the Lienard equation, Differenc. Uravnen. 30 N3 (1994), 1148-1155. 
[31] V. V. Filippov, On the theorem of uniqueness of the solution of the Cauchy problem for ordinary differential equation, Differen. Uravnen., 30 N6 (1994), 1005-1009.

[32] V. V. Filippov, On ordinary differential equations with discontinuities in right-hand sides, Differen. Uravnen. 30 N10 (1994).

[33] Ph. Hartman, Ordinary differential equations, Wiley, 1964.

[34] B. S. Klebanov, On Ważewski's topological principle, Interim report of the Prague Topological Symposium, 2 (1987), 29.

[35] B. S. Klebanov, On $\omega$-limit sets of nonautonomous differential equations, Comment. Math. Uni Carolinae 35, N2 (1994), 267-281.

[36] Cz. Kluczny, Sur certaines familles de courbes en relation avec la théorie des équations différentielles ordinaires, Annales Universitatis M. Curie-Skłodowska, Sec. A., Math. t. XV (1961), 13-40; t. XVI (1962), 5-18.

[37] H. Kneser, Ueber die Lösungen eines gewöhnlicher Differentialgleichungen das der Lipschitzchen Bedingung nicht genügt, S.-P. Preuss. Akad. Wiss. Phys.-Math. Kl. (1923), 171-174.

[38] K. Kuratowski, Sur l'espace des fonctions partielles, Ann. di Mat. Pura ed Appl., 40 (1955) 61-67.

[39] K. Kuratowski, Sur une méthode de métrisation complète, Fund. Math. 43 (1956), 114-138.

[40] K. Kuratowski, Topology, 1, Academic Press, New York - London, PWN, Warszawa, 1966, 2, 1968.

[41] P. N. Savel'ev, On the Poincaré-Bendixson theorem and dissipativity in the plane, Vestnik Moskov. Univers. Ser.1, Math. Mech. N3 (1991), 54-55.

[42] J. Yorke, Spaces of solutions, Mathematical Systems Theory and Economics II, Lecture Notes in Operat. Res. and Math. Econom., Springer-Verlag, 12 (1969), 383-403.

[43] S. K. Zaremba, Sur certaines familles de courbes en relation avec la théorie des équations différentielles, Rocznik Polskiego Tow. Matemat. t. XV (1936), 83-100. 